\title{
Evaluation of annexin A5 as a biomarker for Alzheimer's disease and dementia with lewy bodies
}

\author{
Hitoshi Sohma ${ }^{1,2 *}$, Shin-ichi Imai ${ }^{2}$, Norio Takei ${ }^{2}$, Hirohito Honda ${ }^{3}$, Kyoichi Matsumoto ${ }^{4}$, \\ Kumiko Utsumi ${ }^{5}$, Kayo Matsuki ${ }^{2}$, Eri Hashimoto ${ }^{6}$, Toshikazu Saito ${ }^{6}$ and Yasuo Kokai ${ }^{2}$ \\ 1 Department of Educational Development, Center for Medical Education, Sapporo Medical University, Sapporo, Japan \\ 2 Department of Biomedical Engineering, School of Medicine, Sapporo Medical University, Sapporo, Japan \\ ${ }^{3}$ Mikuri Immunology Laboratory, Inc., Osaka, Japan \\ ${ }^{4}$ Sanyo Chemical Industries, Ltd., Kyoto, Japan \\ ${ }^{5}$ Department of Neuropsychiatry, Sunagawa City Medical Center, Sunagawa, Japan \\ ${ }^{6}$ Department of Neuropsychiatry, School of Medicine, Sapporo Medical University, Sapporo, Japan
}

\section{Edited by:}

Manuel Menéndez-González,

Hospital Álvarez-Buylla, Spain

Reviewed by:

Daniel Ortuño-Sahagun, Centro Universitario de Ciencias Biológicas

y Agropecuarias, Mexico

Junming Wang, University of

Mississippi Medical Center, USA

*Correspondence:

Hitoshi Sohma, Department of

Educational Development, Center

for Medical Education, Sapporo

Medical University, South-1,

West-17, Chuo-ku, Sapporo

060-8556, Japan.

e-mail:sohma@sapmed.ac.jp
Background: Alzheimer's disease (AD) differs from other forms of dementia in its relation to amyloid beta peptide $\left(A \beta_{42}\right)$. Using a cell culture model we previously identified annexin $\mathrm{A} 5, \mathrm{a} \mathrm{Ca}^{2+}$, and phospholipid binding protein, as an $\mathrm{AD}$ biomarker. Plasma level of annexin A5 was significantly higher in $A D$ patients compared to that in a control group. On the other hand, AD has been identified to share a number of clinical and pathological features with Dementia with Lewy bodies (DLB). The present study was done to examine whether or not plasma annexin $A 5$ is a specific marker for $A D$, when being compared with the levels of DLB patients. As Apolipoprotein E (ApoE) gene subtype $\varepsilon 4$ (ApoE- $-\varepsilon 4)$ has been noticed as the probable genetic factor for $A D$, we also examined and compared ApoE genotype in both $A D$ and DLB.

Methods: Blood samples were obtained from 150 patients with $A D$ laged $77.6 \pm 6.5$ years), 50 patients of DLB $(79.4 \pm 5.0)$ and 279 community-dwelling healthy elderly individuals of comparable age and sex $(75.6 \pm 8.1)$. All AD patients met NINCDS-ADRDA criteria and all DLB patients were diagnosed as probable DLB according to the latest consensus diagnostic criteria. Quantification was done using the Chemiluminescent Enzyme Immunoassay (CLEIA) Technique (SphereLight assay) using the monoclonal antibodies against annexin A5. DNA genotyping of ApoE was performed by distinguishing unique combinations of Hha1 fragments of PCR-amplified genomic DNA products.

Results: The plasma level of annexin $A 5$ was significantly higher in $A D$ patients than in the healthy individuals (control) $(P<0.0001)$. The plasma annexin $A 5$ level was also significantly higher in DLB patients than in the control group $(P<0.0001)$. From the ROC curves with plasma annexin $\mathrm{A} 5$ concentrations, the mean areas under the curve were 0.863 and 0.838 for the $\mathrm{AD} /$ control and $\mathrm{DLB} /$ control, respectively. The rate of ApoE4 carrier status and the frequency of the $\varepsilon 4$ allele were significantly higher in AD or DLB than in control and there was no significant difference between AD and DLB.

Conclusions: These results suggest that both annexin A5 and ApoE4 are common markers for $A D$ and $D L B$.

Keywords: plasma biomarker, Alzheimer's disease, dementia with lewy bodies, annexin A5, $\mathrm{Ca}^{2+}$-stress, ROC curve, ApoE

\section{INTRODUCTION}

The augmented number of dementia patients is remarkable in the aging of society in advanced countries. Alzheimer's disease (AD) accounts for more than half of all dementia, and Dementia with Lewy bodies (DLB) are the second most common, accounting for approximately $15 \%$ of cases at autopsy (McKeith et al., 2004), both of which are common forms of neurodegenerative dementia. DLB shares clinical and pathological features with other dementia subtypes such as $\mathrm{AD}$, vascular dementia and Parkinson's disease (PD), which makes it difficult to distinguish in clinical practice. Also, the lack of valid and reliable methods for assessing the core clinical symptoms of both $\mathrm{AD}$ and DLB makes its identification even more difficult. The diagnosis of $\mathrm{AD}$ is reliant on the use of National Institute of Neurological and Communicative Disorders and Stroke-AD and related Disorders Association (NINCDS-ADRDA) criteria. The NINCDS-ADRDA criteria have high sensitivity (0.93), but low specificity (0.23) in the diagnosis of $\mathrm{AD}$ among a group of patients with cortical dementias [AD and frontotemporal dementia (FTD)] (Varma et al., 1999). On the other hand, consensus 
criteria for DLB were developed in 1996 to assist with the antemortem diagnosis (McKeith et al., 2005). Although the criteria have high specificity (79-100\%), the sensitivity is low (20-60\%), so that the diagnosis can be missed in many cases during life (Litvan et al., 2003). The revised clinical consensus criteria were published in 2005, which gives greater diagnostic weight to clinical features suggestive of DLB (McKeith et al., 2005). In light of the limited sensitivity of current methods of clinical diagnosis, it is important to establish additional markers that can improve diagnostic accuracy in combination with clinical assessment.

Amyloid $\beta$ peptide $(A \beta)$, which is a proteolytic product of amyloid precursor protein (APP), accumulates in the brains of $\mathrm{AD}$ patients. Its toxicity is thought to cause neural cell death (Mattson, 2004). Amyloid-dependent neurotoxicity is known to perturb $\mathrm{Ca}^{2+}$ homeostasis in neuronal cells (LaFerla, 2002). Possibly, $\mathrm{A} \beta$ impairs membrane $\mathrm{Ca}^{2+}$ pumps and enhances $\mathrm{Ca}^{2+}$ influx through voltage-dependent channels and ionotropic glutamate receptors. Focusing on this mechanism, we identified the $\mathrm{Ca}^{2+}$-related protein as a potential biomarker for AD using primary neurons as a cell culture model (Yamaguchi et al., 2010). It was shown that the level of annexin A5 was augmented in both the brain and blood plasma in an AD-model mouse (Tg2576 transgenic mouse), overexpressing mutant human APP (Yamaguchi et al., 2010). In addition, the plasma level of annexin A5 was significantly increased in $\mathrm{AD}$ patients compared to that in a control group ( $p$-value of less than 0.0001 in the logistic regression analysis), suggesting that annexin A5 is a favorable marker for AD (Yamaguchi et al., 2010). As annexin A5 binds both $\mathrm{Ca}^{2+}$ and lipids, it might have a role to protect against $\mathrm{Ca}^{2+}$-induced damage. A defensive role against apoptosis by annexin A5 is also reported, in that annexin A5 plays a role in interacting with and reducing the toxicity of the amyloidogenic proteins, islet amyloid polypeptides and $\alpha$-synuclein inclusion (Bedrood et al., 2009).

Apolipoprotein E (ApoE), which is a major component of lipoproteins, is comprised of 299 amino acid residues and plays a role in the metabolism and redistribution of cholesterol. ApoE mediates the uptake of lipoprotein particles in the brain via the low-density lipoprotein (LDL), receptor related protein (LRP), and the very low-density family lipoprotein receptor (VLDL) (Mahley, 1988; Paolo and Kim, 2011). The three major isoforms of ApoE, referred to as ApoE2, E3, and E4, are products of three alleles $(\varepsilon 2, \varepsilon 3, \varepsilon 4)$ at a single gene locus (Mahley, 1988). Three homozygous phenotypes (Apo-E2/2, E3/3, and E4/4) and three heterozygous phenotypes (Apo-E2/3, E3/4, and E2/4) arise from the expression of any two of the three alleles. The $\varepsilon 4$ allele of the ApoE gene was identified as the strongest genetic risk factor for AD (Bertram and Tanzi, 2008). Neuropathological studies demonstrated that the frequency of the ApoE gene subtype $\varepsilon 4$ (ApoE $\varepsilon 4$ ) allele in DLB is similar to AD and that ApoE4 has also been implicated in the development of DLB (Singletona et al., 2002). We reported that ApoE4 genotypes were similar in $\mathrm{AD}$ and $\mathrm{DLB}$, giving further evidence that the $\varepsilon 4$ allele is a risk factor for both disorders in Japanese subjects (Kobayashi et al., 2011).

The present study was done to examine whether or not plasma annexin $\mathrm{A} 5$ is a specific marker for $\mathrm{AD}$, in comparison with the levels of DLB patients. For that purpose, we analyzed plasma level of DLB patients and compared with those of AD patients and agematched community dwelling healthy persons as a control. We further discuss taking ApoE4 frequencies into consideration.

\section{MATERIALS AND METHODS HUMAN BLOOD PLASMA}

The Sapporo Medical University Ethics Committee approved human plasma studies on dementia biomarker study in 2007. Informed written consent was obtained from all subjects. All healthy volunteers and patients provided written permission. For patients with impaired cognition we obtained written permission from their family in accordance with the Declaration of Helsinki. Blood samples were obtained from 150 patients with AD (aged $77.6 \pm 6.5$ years), 50 patients of DLB (aged $79.4 \pm 5.0$ years), and 279 community-dwelling elderly individuals (healthy volunteers) of comparable age and sex (75.6 \pm 8.1 years). All AD patients met NINCDS-ADRDA criteria (McKhann et al., 1984) and DLB patients were diagnosed as probable DLB according to the latest consensus diagnostic criteria (McKeith et al., 2005). The patient's clinical symptoms were evaluated using the revised Hasegawa Dementia scale (HDS-R) (Hasegawa, 1983), Mini-Mental State Examination (MMSE), and clinical dementia rating (CDR). The diagnosis of AD was also confirmed in all patients either by brain magnetic resonance imaging or single photon emission computed tomography. Blood was drawn with Venoject II vacuum tubes containing EDTA-Na (final $4.5 \mathrm{mM}$ ) (Terumo, Tokyo, Japan) and the plasma fraction was isolated by centrifugation at $2500 \mathrm{~g}$ for $15 \mathrm{~min}$. This was repeated once to avoid possible cell debris in blood. Blood was centrifuged within $6 \mathrm{~h}$ after sampling. Plasma fractions were stored at $-80^{\circ} \mathrm{C}$ until use.

\section{QUANTIFICATION OF PLASMA LEVEL OF ANNEXIN A5 USING SANDWICH CLEIA (SPHERELIGHT ASSAY)}

Plasma annexin A5 was quantified using the Chemiluminescent Enzyme Immunoassay (CLEIA) Technique (SphereLight assay) as described (Yamaguchi et al., 2010). Briefly, annexin A5 present in the specimen was trapped by a monoclonal antibody (mAb) against annexin A5 (clone No. 23), conjugated to a glass bead and a horseradish peroxidase (HRP)-labeled $\mathrm{mAb}$ against annexin A5 (clone No. 49). Unbound materials were removed by washing. The chemiluminescent reagent consists of a luminol solution that includes a phenol-derivative as an enhancer, to which a hydrogen peroxide solution was added. The HRP in the bound conjugate catalyzes the oxidation of the luminol derivative, producing light. The light signals were read by the Olympus SphereLight 180 fully automated system (Olympus Optical Co., Ltd., Tokyo, Japan). The amount of HRP conjugate bound was directly proportional to the annexin A5 concentration. The required time and volume of the specimens were $20 \mathrm{~min}$ and $40 \mu \mathrm{l}$, respectively, for the SphereLight assay. The detection limit proved to be $0.16 \mathrm{ng} / \mathrm{ml}$ for annexin A5 and this system was useful to quantify plasma annexin A5 within the range of $0.16-20.0 \mathrm{ng} / \mathrm{ml}$. Reproducible data were obtained by intra-assay and inter-assay (data not shown). Because annexin A5 is present in blood cells (Masuda et al., 2004), if a prolonged period of time has passed (longer than $12 \mathrm{~h}$ ) after collecting blood until centrifuging, the plasma annexin A5 level 
increases (data not shown) due to physical damage such as temperature change, osmotic pressure change and so on. To avoid inducible leakage of annexin A5 from blood cells, all the plasma was separated by centrifugation within $6 \mathrm{~h}$ of sampling. The detection limit proved to be $0.16 \mathrm{ng} / \mathrm{ml}$ of annexin $\mathrm{A} 5$ as previously described (Yamaguchi et al., 2010). We also performed a plasma dilution test and reproducibility studies of intra-assay and inter-assay, which confirmed the assay method is reliable (Yamaguchi et al., 2010).

\section{APOLIPOPROTEIN E (ApoE) GENOTYPING}

DNA genotyping of ApoE was performed according to the protocol described by Hixson and Vernier (1990). Briefly, using a QIAamp DNA Blood Mini Kit (QIAGEN, Tokyo, Japan), genomic DNA was extracted from the buffy coat after centrifugation of the blood sample according to the manufacturer's instructions. The leukocyte DNA was amplified by PCR using the oligonucleotide primers, Primer 1 (59-TAAGCTTGGCACGGCTGTCCAAGGA39), and Primer 2 (59-ACAGAATTCGCCCCGGCCTGGTACAC39) set on common sequence parts of ApoE isoforms. The PCR products were digested with HhaI (New England Biolabs, Japan, Inc., Tokyo, Japan) and the resulting digestion fragments were separated by electrophoresis on polyacrylamide gels (SuperSepTMDNA 15\% gel (Wako, Tokyo, Japan)). Each genotype of ApoE was distinguished by unique combinations of Hhal fragment sizes in all homozygotic and heterozygotic combinations (Hixson and Vernier, 1990). After determining the ApoE genotypes, we investigated the ApoE4 carrier status and the frequency of the $\varepsilon 4$ allele in the 279 controls, $150 \mathrm{AD}$, and $50 \mathrm{DLB}$ cases.

\section{STATISTICAL ANALYSIS}

The mean response of each experimental group was compared with its simultaneous control by the unpaired Student's $t$-test. Analysis of variance was used to compare the mean responses of the experimental and control groups. A significant difference was set at $p<0.05$. Logistic regression modeling was employed to construct receiver operator curves (ROC) by using JMP 9.0.0 (SAS Institute Inc., Cary, NC) to examine the plasma annexin A5 levels in diagnoses of $\mathrm{AD}$ and DLB. ROC curve comparisons were based on the area under the curve (AUC), SE, and the associated 95\% confidence interval (CI). We subsequently calculated sensitivity of the various models using the predicted probability of each subject by logistic regression modeling with specificity of at least eighty percent. Fisher's exact tests were used to assess the frequencies of the $\varepsilon 4$ allele between groups using JMP 9.0.0 (SAS Institute, Cary, NC, USA).

\section{RESULTS}

Plasma level of annexin A5 was analyzed using CLEIA Technique (SphereLight assay) as described in Materials and Methods. In this study, we measured 150 samples of $\mathrm{AD}$ (age $77.6 \pm 6.5$ ), 50 samples of DLB (age $79.4 \pm 5.0$ ), and 279 age-matched community dwelling healthy persons (age $75.6 \pm 8.1$ ) as a control. When average concentrations of plasma annexin A5 are compared among $\mathrm{AD}, \mathrm{DLB}$, and control groups, the values of $\mathrm{AD}(3.33 \pm 1.60)$ and DLB $(3.02 \pm 1.08)$ were significantly higher than healthy control

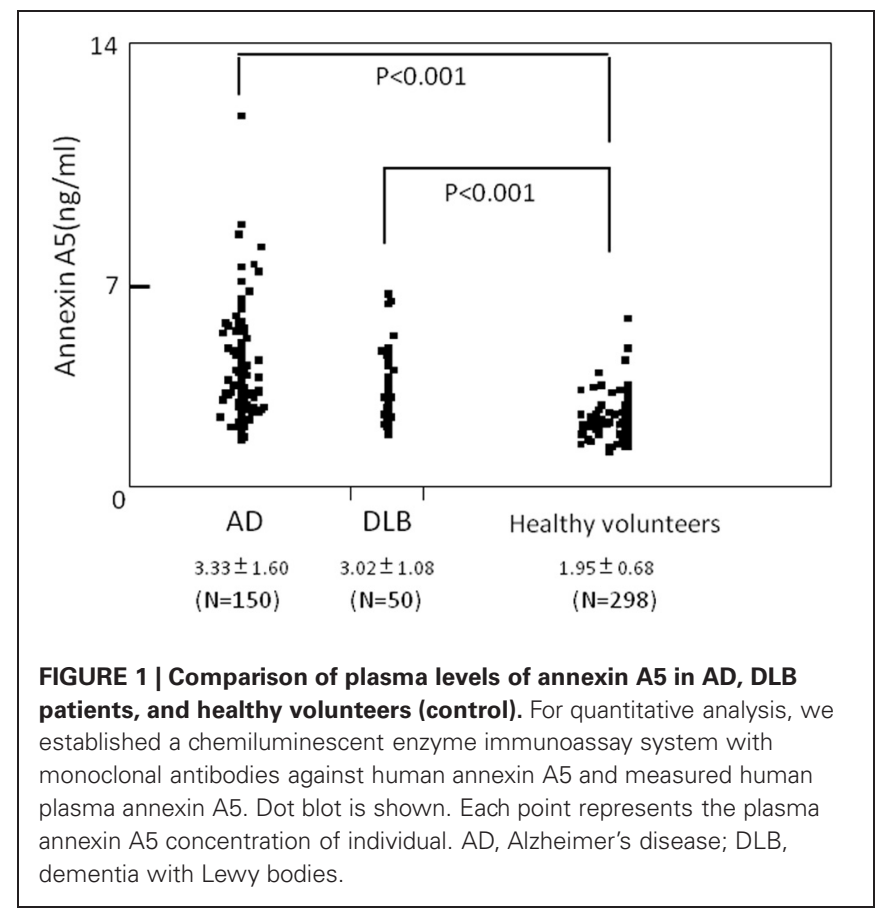

subjects $(1.95 \pm 0.68)$ (Figure 1). The probability of both AD and DLB can be predicted by a logistic regression model with the plasma level of annexin A5. The ROC analyses revealed good separation of patients with either AD or DLB from healthy control subjects (Figure 2). The areas under the curve were $86.3 \%$ $(P<0.0001)$ and $83.8 \%(P<0.0001)$ for $\mathrm{AD}$ and DLB, respectively. That is statistically significant, suggesting that annexin A5 is also a potential biomarker for both $\mathrm{AD}$ and DLB. On the other hand, no significant difference was observed between $\mathrm{AD}$ and $\operatorname{DLB}(p=0.36)$.

Several risk factors for $\mathrm{AD}$ have been suggested such as medical history, life style, environment and genes. Of these, ApoE- $\varepsilon 4$ has been noticed as one of the genetic factors. We next identified ApoE gene typing by analyzing the restriction enzyme products of the PCR-amplified ApoE gene as shown in Materials and Methods (Table 1, Figure 3). In the control group, 51 out of 279 subjects were ApoE4 carriers (18.3\%). Three subjects were homozygous for the $\varepsilon 4$ allele $(1.1 \%)$ and 48 subjects were heterozygous for the e4 allele (17.2\%). The total frequency of the $\varepsilon 4$ allele was $9.7 \%$. In the $\mathrm{AD}$ group, 63 out of 150 subjects were ApoE4 carriers $(42.0 \%)$. Nine subjects were homozygous for the $\varepsilon 4$ allele $(6.0 \%)$ and 54 subjects were heterozygous for the $\varepsilon 4$ allele $(36.0 \%)$. The total frequency of the $\varepsilon 4$ allele was $24.0 \%$. In the DLB group, 21 out of 50 subjects were ApoE4 carriers (42.0\%). Three subjects were homozygous for the $\varepsilon 4$ allele $(6.0 \%)$ and 18 subjects were heterozygous for the $\varepsilon 4$ allele $(36.0 \%)$. The total frequency of the $\varepsilon 4$ allele was $24.0 \%$. ApoE4 frequencies were compared among AD, DLB, and control groups (Fisher's exact test). ApoE4 carrier status was significantly different between $\mathrm{AD}$ and control groups $(p<0.0001)$, and between DLB and control $(p=$ $0.0004)$. Allele frequencies of ApoE $\varepsilon 4$ were significantly higher in $\mathrm{AD}(p<0.0001)$ and $\mathrm{DLB}(p<0.0001)$ than in the control 


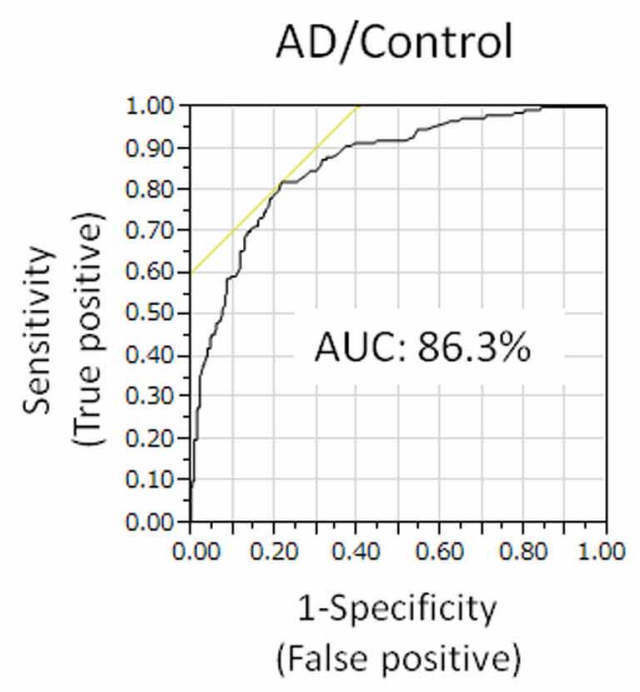

FIGURE 2 | Receiver operating characteristic (ROC) curves showing prediction of either AD or DLB by plasma annexin A5. The probability of either $A D$ or DLB can be predicted by a logistic regression model with the

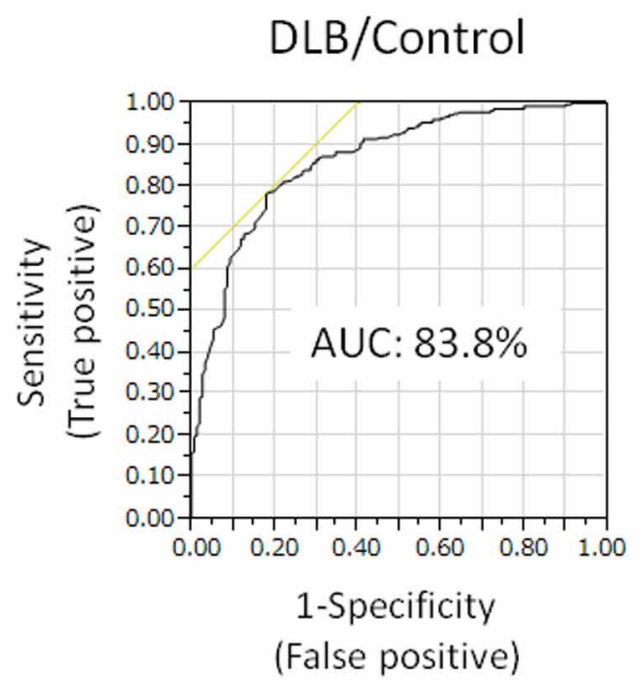

plasma level of annexin A5. The areas under the curve are 86.3 and $83.8 \%$ for $A D$ and $D L B$, respectively. AD, Alzheimer's disease; DLB, dementia with Lewy bodies.
Table 1 | Distribution of ApoE4 carrier status and the frequency of ApoE $\varepsilon 4$ allele in the population of $A D, D L B$, and control groups.

\begin{tabular}{llrllc}
\hline & \multicolumn{2}{c}{ ApoE4 carrier* } & & \multicolumn{2}{c}{ ApoE \&4 allele** } \\
\cline { 2 - 3 } \cline { 5 - 6 } & Positive & Negative & & Positive & Negative \\
\hline C & $51(18.3 \%)$ & $228(81.7 \%)$ & & $57(10.2 \%)$ & $501(89.8 \%)$ \\
AD & $63(42.0 \%)$ & $87(58.0 \%)$ & & $72(24.0 \%)$ & $228(76 \%)$ \\
DLB & $21(42.0 \%)$ & $29(58.0 \%)$ & & $27(24.0 \%)$ & $73(76.0 \%)$ \\
\hline
\end{tabular}

Numbers in parentheses represent the frequencies of ApoE4 carrier or ApoE $\varepsilon 4$ allele. $C$, control group; $A D$, Alzheimer's disease group; $D L B$, dementia with Lewy bodies group.

*Significantly different between $A D$ and control groups $(p<0.0001)$, and between DLB and control ( $p=0.0004)$.

** Significantly higher in AD ( $p<0.0001)$ and DLB $(p<0.0001)$ than in the control group. No significant differences in rates of ApoE4 carrier status and the frequencies of the $\varepsilon 4$ allele between $A D$ and $D L B$.

group. However, there were no significant differences in rates of ApoE4 carrier status $(p=0.57)$ and the frequencies of the $\varepsilon 4$ allele $(p=0.32)$ between $\mathrm{AD}$ and DLB. These results also indicate the similarity of $\mathrm{AD}$ and DLB.

\section{DISCUSSION}

\section{SIMILARITY OF AD AND DLB}

The toxicity of $A \beta$ is thought to cause neural cell death, which is involved in the pathogenesis AD (Mattson, 2004). Decreased degradation or dyscatabolism of $\mathrm{A} \beta$, presumably related to aging, results in both the accumulation of amyloid beta peptide $\left(\mathrm{A} \beta_{42}\right)$ in the brain and the decreased concentration of $A \beta_{42}$ in CSF. Thus, lowered concentration of CSF $A \beta_{42}$ has been noted as a barometer for $\mathrm{AD}$ (Andreasen et al., 2001). AD is the most common neurodegenerative dementia and DLB is the second most common. DLB shares clinical and pathological features with $\mathrm{AD}$, which makes it difficult to distinguish in clinical practice. The CSF levels of $A \beta_{42}$ are similar between $A D$ and DLB (Gomez-Tortosa et al., 2003; Mollenhauer et al., 2005a). Amyloiddependent neurotoxicity is known to perturb $\mathrm{Ca}^{2+}$ homeostasis in neuronal cells (LaFerla, 2002). Possibly, A $\beta$ impairs membrane $\mathrm{Ca}^{2+}$ pumps and enhances $\mathrm{Ca}^{2+}$ influx through voltage-dependent channels and ionotropic glutamate receptors. Focusing on proteins concerning $\mathrm{Ca}^{2+}$ signaling, we identified annexin $\mathrm{A} 5$ which is augmented in $\mathrm{A} \beta_{42}$ dependent manner and showed it as a potential biomarker for AD (Yamaguchi et al., 2010). Moreover, the plasma level of annexin A5 was shown to be elevated in AD (Yamaguchi et al., 2010). In the present study, plasma level of annexin A5 was shown to be elevated not only in AD but also in DLB.

Genetic factors are increasingly recognized as major risk factors for dementia. Evidence from numerous studies has identified the ApoE gene on chromosome 19 as a major risk factor for AD. ApoE, which is a major component of lipoproteins, is comprised of 299 amino acid residues and plays a role in the metabolism and redistribution of cholesterol (Hatters et al., 2006). Three major common isoforms, designated ApoE2, ApoE3, and ApoE4. ApoE colocalizes with extracellular amyloid deposits, resulting in isoform-specific clearance of $A \beta$. However, ApoE isoforms differently interact with $A \beta$ isoform specific effects on $A \beta$-clearance. In ApoE4, domain interaction occurs as a result of a putative salt bridge, leading to tight structural formation. This interaction does not occur to the same extent in ApoE2 and ApoE3 (Dong et al., 1994; Dong and Weisgraber, 1996). ApoE $\varepsilon 4$ is associated with an increased risk for $\mathrm{AD}$ with an earlier age of disease onset (Kim et al., 2009). On the other hand, findings regarding ApoE polymorphisms in DLB have so far been inconclusive. It was 

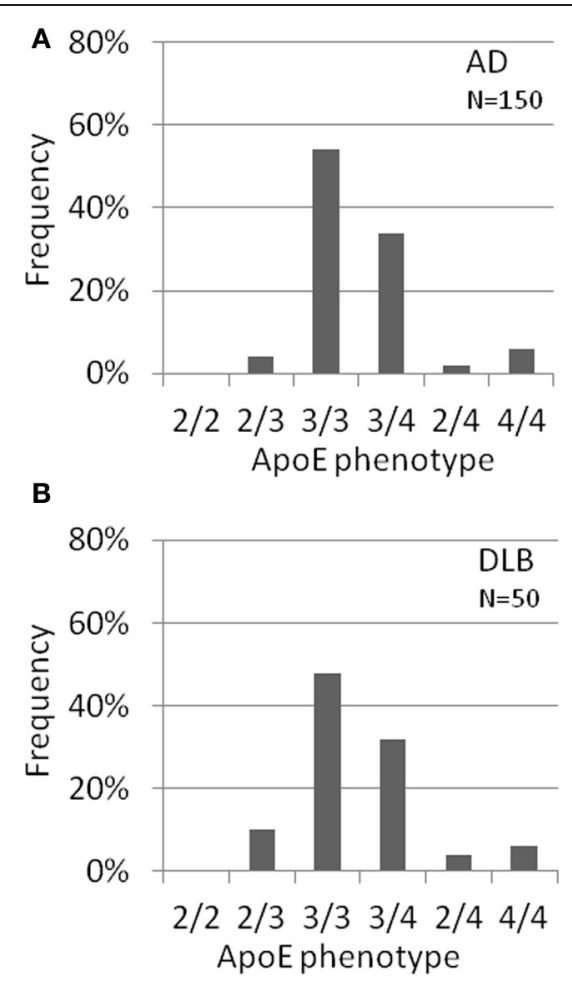

FIGURE 3 | ApoE carrier statuses of AD (A), DLB (B), and healthy volunteers (control) (C). ApoE carrier statuses are significantly different between $A D$ and control, and between DLB and control. However, no

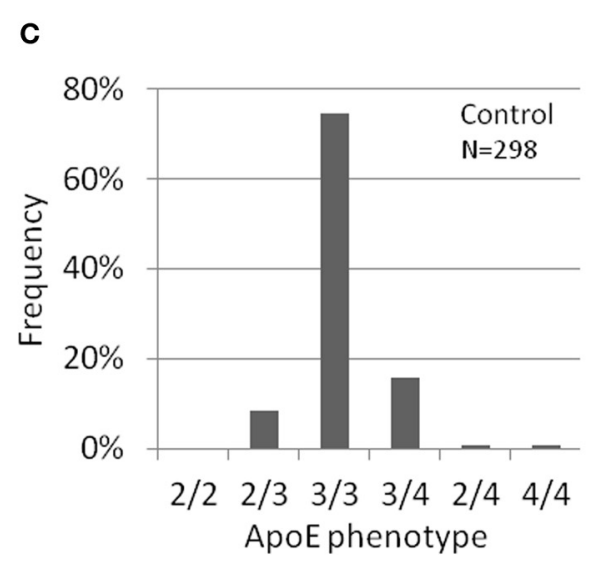

significant differences were observed between AD and DLB. ApoE typing was done using pcr and restriction enzyme digestion using genomic DNA. AD, Alzheimer's disease; DLB, dementia with Lewy bodies. reported that ApoE4 carrier frequency was the highest in $\mathrm{AD}$ among $\mathrm{AD}, \mathrm{DLB}$, and control groups, and it was higher in DLB than in control groups (Carrillo Garcia et al., 2008). Other findings have shown that ApoE4 carrier and allelic frequencies were comparable for those with AD and DLB [(Kobayashi et al., 2011) and Table 1 ].

Our results for annexin A5 and ApoE4 also revealed similar characteristics for both $\mathrm{AD}$ and DLB patients.

\section{DIFFERENCE BETWEEN AD AND DLB}

It is apparent that DLB differs from AD in the disease progression and cure response experienced by patients. Accordingly, early differentiation between the two forms of dementia is important for effective and safe management (Aarsland et al., 2008; Sinha et al., 2011). CSF levels of tau protein have been shown to be significantly lower in DLB than in $\mathrm{AD}$, which may help to differentiate between the two diseases (Mollenhauer et al., $2005 a, b)$. On the other hand, another study also suggests that the concentration of phosphorylated tau in CSF, which is highly correlated with total tau levels, may provide a higher specificity to differentiate AD and DLB (Vanderstichele et al., 2006). $\alpha$-Synuclein is the major constituent of Lewy bodies found in neurons in DLB. As a consequence of increased accumulation of $\alpha$-synuclein intraneuronally in DLB, several studies have attempted its quantification in CSF. $\alpha$-Synuclein has been shown to induce disruption of cellular inorganic ion homeostasis such as $\mathrm{Ca}^{2+}$, leading to cell death (Lowe et al., 2004; Danzer et al., 2007; Ying et al., 2011). Whereas some groups show a decrease in the total concentration of CSF $\alpha$-synuclein in DLB in comparison to other dementias (Mollenhauer et al., 2008; Kasuga et al., 2010), other groups do not find the significant difference for DLB (Spies et al., 1998; Noguchi-Shinohara et al., 2009). Thus, future study on the discrimination of these diseases is expected.

\section{ABOUT BIOMARKERS}

One of the main focuses of public health is prevention of disease. Different stages in the disease process can be targeted for preventative action, including prior to development of the disease, during the asymptomatic stage, and following clinical diagnosis. Therefore, three stages of prevention can be recognized (Wright et al., 2009; Weber et al., 2012).

From the CSF proteins identification, and MRI and PET imaging studies, the alteration of both CSF biomarkers ( $\mathrm{A} \beta_{42}$ and Tau) takes place prior to the appearance of brain structural change or dementia symptoms (Jack et al., 2010). Our in vitro data demonstrated that annexin A5 is elevated following the stimulation by $\mathrm{A} \beta_{42}$ (Yamaguchi et al., 2010). Thus, onset of the annexin A5 elevation in dementia occurs at the similar time to the deposition of $\mathrm{A} \beta_{42}$. Annexin $\mathrm{A} 5$ might be expected to be useful in the secondary and tertiary stages. It is conceivable that the appropriate stage for utilizing each biomarker candidate is dependent upon the properties of the biomarker. Therefore, to determine when each biomarker candidate should be utilized it will be necessary 
to examine the significance of any biological changes that appear at various stages.

Discrimination between neurodegenerative and nonneurodegenerative dementia is another expectation for biomarkers. Shared clinical symptoms between $\mathrm{AD}$ and depression in elderly have been reported (Starkstein et al., 2005), which might lead to confusion in medical intervention. Our preliminary data suggest that plasma annexin A5 levels of the six patients with depression was comparable with controls (data not shown), which might implicate annexin A5 as a biomarker for discriminating between neurodegenerative and non-neurodegenerative diseases.

Biomarkers should be reliable, reproducible, non-invasive, simple to perform, and inexpensive. To achieve this role both protein-based and genetic biomarkers have been particularly investigated. Especially plasma biomarker is beneficial by being

\section{REFERENCES}

Aarsland, D., Kurz, M., Beyer, M., Bronnick, K., Nore, S. P., and Ballard, C. (2008). Early discriminatory diagnosis of dementia with Lewy bodies. Dement. Geriatr. Cogn. Disord. 25, 195-205.

Andreasen, N., Minthon, L., Davidsson, P., Vanmechelen, E., Vanderstichele, H., Winblad, B., et al. (2001). Elevation of CSF-tau and CSF$\mathrm{A} \beta 42$ as diagnostic markers for Alzheimer disease in clinical practice. Arch. Neurol. 58, 373-379.

Bedrood, S. S., Jayasinghe, D., Sieburth, M., Chen, S., Erbel, P. C., Butler, R., et al. (2009). Annexin A5 direcly interacts with amyloidogenic proteins and reduces their toxicity. Biochemistry 48, 10568-10576.

Bertram, L., and Tanzi, R. E. (2008). Thirty years of Alzheimer's disease genetics: the implications of systematic meta-analysis. Nat. Rev. Neurosci. 9, 768-778.

Carrillo Garcia, F., Gil Neciga, E., Alberca, R., Garcia-Solis, D., Millan, J., Rodriguez Uranga, J. J., et al. (2008). Apolipoprotein $\varepsilon 4$ in dementia with Lewy bodies. Neurologia 23, 152-156.

Danzer, K. M., Haasen, D., Karow, A. R., Moussaud, S., Habeck, M., Giese, A., et al. (2007). Different species of alpha-synuclein oligomers induce calcium influx and seeding. J. Neurosci. 27, 9220-9232.

Dong, L.-M., and Weisgraber, K. H. (1996). Human apolipoprotein E4 domain interaction. Arginine 61 and glutamic acid 255 interact to direct the preference for very low density lipoproteins. J. Biol. Chem. 271, 19053-19057.
Dong, L.-M., Wilson, C., Wardell, M. R., Simmons, T., Mahley, R. W., Weisgraber, K. H., et al. (1994). Human apolipoprotein E. Role of arginine 61 in mediating the and E4 isoforms. J. Biol. Chem. 269, 22358-22365.

Gomez-Tortosa, E., Gonzalo, I., Fanjul, S., Sainz, M. J., Cantarero, S., Cemillan, C., et al. (2003). Cerebrospinal fluid markers in dementia with Lewy bodies compared with Alzheimer disease. Arch. Neurol. 60, 1218-1222.

Hasegawa, K. (1983). "The clinical assessment of dementia in the aged: a dementia screening scale for grading the cognitive state of patients for clinician," in Aging in the Eighties and Beyond, ed M. E. A. Bergener (New York, NY: Springer), 207-218.

Hatters, D. M., Peters-Libeu, C. A., and Weisgraber, K. H. (2006). Apolipoprotein E structure: insights into function. Trends Biochem. Sci. 31, 445-454.

Hixson, J. E., and Vernier, D. T. (1990). Restriction isotyping of human apolipoprotein E by gene amplification and cleavage with HhaI. J. Lipid Res. 31, 545-548.

Jack, C. R. Jr., Knopman, D. S., Jagust, W. J., Shaw, L. M., Aisen, P. S., Weiner, M. W., et al. (2010). Hypothetical model of dynamic biomarkers of the Alzheimer's pathological cascade. Lancet Neurol. 9, 119-128.

Kasuga, K., Tokutake, T., Ishikawa, A., Uchiyama, T., Tokuda, T., Onodera, O., et al. (2010). Differential levels of $\alpha$-synuclein, $\beta$-amyloid 42 and tau in CSF between patients with dementia with Lewy bodies and pipoprotein preferences of the E3

less invasive in comparison with CSF biomarker. Gene typing is also less invasive since it is available with leukocytes from a blood sample and genetic biomarkers are of great use. ApoE4 $\varepsilon 4$ is widely recognized as a potential biomarker for the risk of $\mathrm{AD}$. As we demonstrated in this paper, ApoE $\varepsilon 4$ is also a risk factor for $\mathrm{DLB}$, indicating that $\mathrm{ApoE} \varepsilon 4$ is unable to discriminate between $\mathrm{AD}$ and DLB. No applicable genetic marker for such purpose has been reported. Detailed molecular mechanism of the onset of both AD and DLB may be needed to explore genetic factors.

\section{ACKNOWLEDGMENTS}

We thank Mr. Kenneth E. Hartman for critical reading of the manuscript. This study was supported by grants from the Ministry of Health, Labor, and Welfare of Japan and from the Ministry of Education, Culture, Sports, Science, and Technology of Japan (grant number 24591686).

Alzheimer's disease. J. Neurol. Neurosurg. Psychiatry 81, 608-610.

Kim, J., Basak, J. M., and Holtzman, D. M. (2009). The role of apolipoprotein $\mathrm{E}$ in Alzheimer's disease. Neuron 63, 287-303.

Kobayashi, S., Tateno, M., Park, T. W. Utsumi, K., Sohma, H., Ito, Y. M., et al. (2011). Apolipoprotein E4 frequencies in a Japanese population with Alzheimer's disease and dementia with Lewy bodies. PLoS ONE 6:e18569. doi: 10.1371/journal.pone.0018569

LaFerla, F. M. (2002). Calcium dyshomeostasis and intracellular signalling in Alzheimer's disease. Nat. Rev. Neurosci. 3, 862-872.

Litvan, I., Bhatia, K. P., Burn, D. J., Goetz, C. G., Lang, A. E., McKeith, I., et al. (2003). SIC task force appraisal of clinical diagnostic criteria for Parkinsonian disorders. Mov. Disord. 18, 467-486.

Lowe, R., Pountney, D. L., Jensen, P. H., Gai, W. P., and Voelcker, N. (2004). Calcium (II) selectively induces $\alpha$ synuclein annular oligomers via interaction with the C-terminal domain. Protein Sci. 13, 3245-3252.

Mahley, R. W. (1988). Apolipoprotein E: cholesterol transport protein with expanding role in cell biology. Science 240, 622-629.

Masuda, J., Takayama, E., Satoh, A., Ida, M., Shinohara, T., Kojima-Aikawa, K., et al. (2004). Levels of annexin $\mathrm{IV}$ and $\mathrm{V}$ in the plasma of pregnant and postpartum women. Thromb. Haemost. 91, 1129-1136.

Mattson, M. P. (2004). Pathways towards and away from Alzheimer's disease. Nature 430, 631-639.

McKeith, I., Mintzer, J., Aarsland, D., Burn, D., Chiu, H., CohenMansfield, J., et al. (2004).
Dementia with Lewy bodies. Lancet Neurol. 3, 19-28.

McKeith, I. G., Dickson, D. W., Lowe, J., Emre, M., O’Brien, J. T., Feldman, H., et al. (2005). Diagnosis and management of dementia with Lewy bodies: third report of the DLB Consortium. Neurology 65, 1863-1872.

McKhann, G., Drachman, D., Folstein, M., Katzman, R., Price, D., and Stadlan, E. M. (1984). Clinical diagnosis of Alzheimer's disease: report of the NINCDS-ADRDA work group under the auspices of department of health and human services task force on Alzheimer's disease. Neurology 34, 939-944.

Mollenhauer, B., Bibl, M., Trenkwalder, C., Stiens, G., Cepek, L., Steinacker, P., et al. (2005a). Follow-up investigation in cerebrospinal fluid of patients with dementia with Lewy bodies and Alzheimer's disease. J. Neural Transm. 112, 933-948.

Mollenhauer, B., Cepek, L., Bibl, M., Wiltfang, J., Schulz-Schaeffer, W. F., Ciesielczyk, B., et al. (2005b). Tau protein, $A \beta 42$ and S-100B protein in cerebrospinal fluid of patients with dementia with Lewy bodies. Dement. Geriatr. Cogn. Disord. 19, 164-170.

Mollenhauer, B., Cullen, V., Kahn, I., Krastins, B., Outeiro, T. F., Pepivani, I., et al. (2008). Direct quantification of CSF alpha-synuclein by ELISA and first cross-sectional study in patients with neurodegeneration. Exp. Neurol. 213, 315-325.

Noguchi-Shinohara, M., Tokuda, T., Yoshita, M., Kasai, T., Ono, K., Nakagawa, M., et al. (2009). CSF alpha synuclein levels in dementia with Lewy bodies and Alzheimer's disease. Brain Res. 1251, 1-6. 
Paolo, G. D., and Kim, T.-W. (2011). Linking lipids to Alzheimer's disease: cholesterol and beyond. Nat. Rev. Neurosci. 12, 284-296.

Singletona, A. B., Whartona, A., O’Briena, K. K., Walker, M. P., McKeith, I. G., Ballard, C. G., et al. (2002). Clinical and neuropathological correlates of apolipoprotein E genotype in Dementia with Lewy Bodies. Dement. Geriatr. Cogn. Disord. 167-175.

Sinha, N., Firbank, M., and O’Brien, J. T. (2011). Biomarkers in dementia with Lewy bodies: a review. Int. J. Geriatr. Psychiatry 27, 443-453.

Spies, C. D., Kissner, M., Neumann, T., Blum, S., Voigt, C., Funk, T., et al. (1998). Elevated carbohydratedeficient transferrin predicts prolonged intensive care unit stay in traumatized men. Alcohol Alcohol. 33, 661-669.

Starkstein, S. E., Jorge, R., Mizrahi, R., and Robinson, R. G. (2005). The construct of minor and major depression in Alzheimer's disease. Am. J. Psychiatry 162, 2086-2093.

Vanderstichele, H., De Vreese, K., Blennow, K., Andreasen, N., Sindic, C., Ivanoiu, A., et al. (2006). Analytical performance and clinical utility of the INNOTEST PHOSPHO-TAU181P assay for discrimination between Alzheimer's disease and dementia with Lewy bodies. Clin. Chem. Lab. Med. 44, 1472-1480.

Varma, A. R., Snowden, J. S., Lloyd, J. J., Talbot, P. R., Mann, D. M. A. and Neary, D. (1999). Evaluation of the NINCDS-ADRDA criteria in the differentiation of Alzheimer's disease and frontotemporal dementia. J. Neurol. Neurosurg. Psychiatry 66, 184-188.

Weber, G. F., Warren, J., Sohma, H., Chen, T., Halim, A. and Chakravarty, G. (2012). Biomarkers-a pot of gold or a can of worms? Meeting report from the 2nd World Congress on
Biomarkers and Clinical Research, 2011, Baltimore, USA. Cancer Biol. Ther. 13, 831-835.

Wright, C. F., Hall, A., Matthews, F. E. and Brayne, C. (2009). Biomarkers, dementia, and public health. Ann. N.Y. Acad. Sci. 1180, 11-19.

Yamaguchi, M., Kokai, Y., Imai, S., Utsumi, K., Matsumoto, K., Honda, H., et al. (2010). Investigation of annexin A5 as a biomarker for Alzheimer's disease using neuronal cell culture and mouse model. J. Neurosci. Res. 88 , 2682-2692.

Ying, Z., Lin, F., Gu, W., Su, Y., Arshad, A., Quuing, H., et al. (2011). $\alpha$-Synuclein increases U251 cells vulnerability to hydrogen peroxide by disrupting calcium homeostasis. J. Neural Transm. 118 , 1165-1172.

Conflict of Interest Statement: The authors declare that the research was conducted in the absence of any commercial or financial relationships that could be construed as a potential conflict of interest.

Received: 23 December 2012; accepted: 19 March 2013; published online: 05 April 2013.

Citation: Sohma H, Imai S-i, Takei $N$, Honda H, Matsumoto K, Utsumi K, Matsuki K, Hashimoto E, Saito T and Kokai Y (2013) Evaluation of annexin A5 as a biomarker for Alzheimer's disease and dementia with lewy bodies. Front. Aging Neurosci. 5:15. doi: 10.3389/fnagi. 2013.00015

Copyright (C) 2013 Sohma, Imai, Takei Honda, Matsumoto, Utsumi, Matsuki, Hashimoto, Saito and Kokai. This is an open-access article distributed under the terms of the Creative Commons Attribution License, which permits use, distribution and reproduction in other forums, provided the original authors and source are credited and subject to any copyright notices concerning any thirdparty graphics etc. 